\title{
Measurement of PDE of MPPC with different wavelengths of light
}

\author{
Daniel Orme*, T.Nakaya, M. Yokoyama, A. Minamino \\ Kyoto Univ. \\ E-mail: danielescphys.kyoto-u.ac.jp
}

\begin{abstract}
We have performed measurements of the Photon Detection Efficiency (PDE) of Multi-Pixel Photon Counters (MPPCs) illuminated with different wavelengths of light. Since photons of different wavelengths will penetrate the silicon layer of the MPPC to different depths, it is expected that the voltage dependence of the PDE may differ with wavelength. The results show that at high bias voltages, the PDE saturates less with long wavelengths of incident light. This result supports the initial predictions, although further quantitative investigation is required to understand the underlying effects.
\end{abstract}

International Workshop on New Photon Detectors

June 24-26, 2009

Shinshu University Matsumoto Japan

${ }^{*}$ Speaker. 


\section{Introduction}

We have investigated the over-voltage $(\Delta V)$ dependence of the Photon Detection Efficiency (PDE) of MPPCs with different wavelengths of incident light. For this purpose we have made several measurements using two types of MPPC: Hamamatsu S10362-13-050P T2K MPPCs and Hamamatsu S10362-11-050P Surface Mount MPPCs [1]. The T2K MPPC has a $1.3 \mathrm{mmx} 1.3 \mathrm{~mm}$ active area and 667 pixels, and a photograph is shown in Figure 1. The Surface Mount MPPC has a $1.0 \mathrm{mmx} 1.0 \mathrm{~mm}$ active area and 400 pixels. In addition it has a thinner silicon structure. The basic properties of these two MPPCs are shown in Table 1.

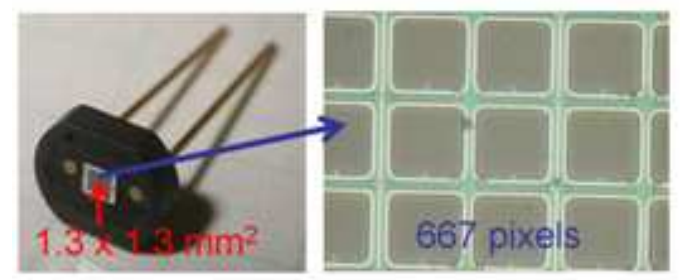

Figure 1: Photograph of $1.3 \mathrm{mmx} 1.3 \mathrm{~mm} \mathrm{~T} 2 \mathrm{~K}$ MPPC. Left: entire device; Right: close-up view of APD pixels

Table 1: Basic properties of Hamamatsu MPPCs. These values are representative of both types of MPPC used[1].

\begin{tabular}{lc} 
Item & Value \\
\hline \hline Pixel size & $50 \times 50 \mu \mathrm{m}^{2}$ \\
Operation voltage & $\sim 70 \mathrm{~V}$ \\
Gain at operation voltage & $7 \times 10^{5}$ \\
Dark count $(>0.5$ p.e. $)$ & $400 \mathrm{kHz}$
\end{tabular}

Each pixel of the MPPC operates essentially as an avalanche photodiode (APD) in Geiger mode. Since each pixel operates in Geiger mode the signal from a single pixel is independent of the number of photons incident on that pixel. In an MPPC many such pixels are connected with a common readout, so the total signal is proportional to the number of pixels fired. For a small number of incident photons compared to the number of pixels, this allows excellent photon counting capability.

\section{Motivation \& Theory}

Figure 2 shows the basic structure of an avalanche photodiode, which has the same operation principle as a single MPPC pixel. The silicon can be divided into 3 layers: the p+ layer, the multiplication layer and the $n+$ layer. An incoming photon creates an electron-hole pair inside the silicon. The electric field inside the silicon causes electrons created in the $\mathrm{p}+$ layer to drift towards the multiplication layer. and holes created in the $n+$ layer to drift towards the multiplication layer. When an electron or hole drifts to the multiplication layer, it triggers a Geiger avalanche which produces a signal with around $10^{5}$ gain. The efficiency of detecting a photon entering the MPPC, i.e. the photon detection efficiency (PDE), is a product of three terms:

$$
P D E=\varepsilon_{\text {geom }} \cdot Q E \cdot \varepsilon_{\text {Geiger }}
$$

1. The geometrical efficiency $\varepsilon_{\text {geom }}$ is simply the ratio of the active area of the MPPC to the total area of the MPPC. This term is a constant for the same MPPC design.

2. The quantum efficiency (QE) of the MPPC is the probability an incoming photon to create an electron-hole pair in the silicon. This term is a function of the wavelength of the incoming photon.

3. The Geiger (or avalanche) efficiency $\varepsilon_{\text {Geiger }}$ is the probability for an electron or hole to trigger a Geiger avalanche in the multiplication layer. This term is a function of the bias voltage applied to the MPPC. 


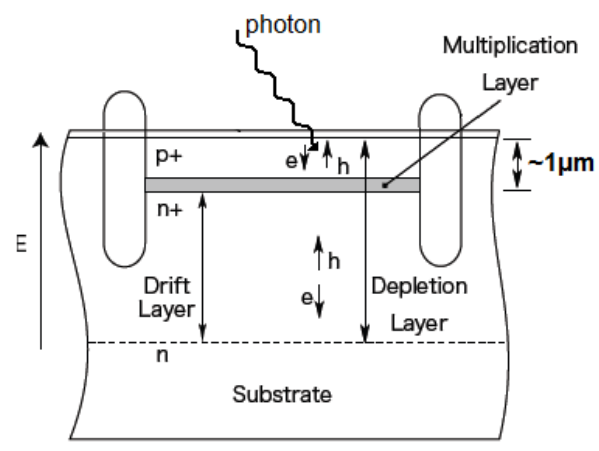

Figure 2: Basic structure of avalanche photodiode (analagous to one pixel of MPPC). Electron-hole pairs created in the $\mathrm{p}+$ silicon result in electron triggered avalanches. Electron-hole pairs created in the $\mathrm{n}+$ silicon result in hole triggered avalanches.

It has been proposed that the Geiger probability has a different dependence on bias voltage for electrons and holes[2]. Figure 3 shows how the Geiger probability is expected to differ. At high bias voltage, the Geiger probability for electrons saturates, but in contrast the Geiger probability for holes remains linear. Since the PDE bias voltage dependence is entirely due to the $\varepsilon_{\text {Geiger }}$ term, the voltage dependence of the PDE will change depending on whether the avalanches are triggered by electrons or holes.

Table 2 shows the mean free path in Silicon of four wavelengths of light, from blue to red. The thickness of the p+ layer in an MPPC is of the order of $1 \mu \mathrm{m}$. It can therefore be seen that the majority of blue photons entering an MPPC will be absorbed within the p+ layer, and therefore the vast majority of avalanches due to blue photons will be triggered by electrons originating in the $\mathrm{p}+$ layer. However, for red light a significant proportion of photons will pass through to the $\mathrm{n}+$ layer. This means that for red light there will be a significant contribution from avalanches triggered by holes originating in the $n+$ layer.

If, as theorised, the PDE dependence on bias voltage is different for avalanches triggered

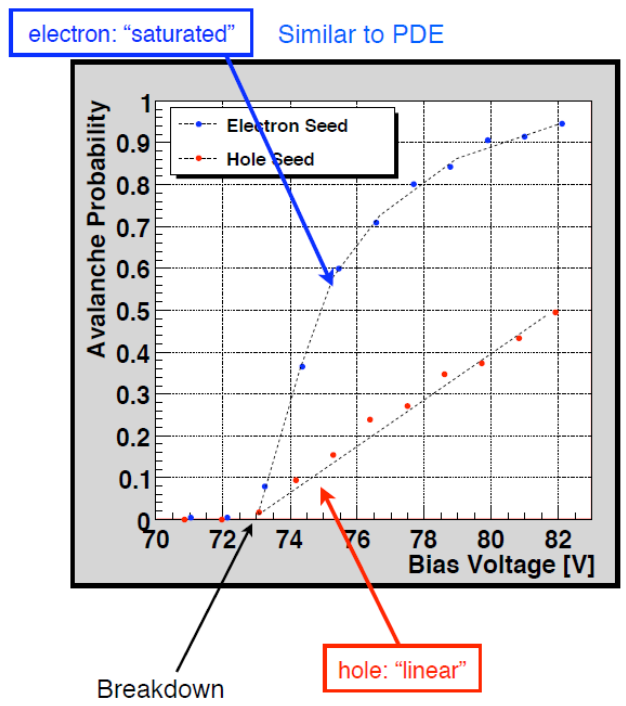

Figure 3: Simulation of avalanche (or Geiger) probability for electrons and holes from [2]. Note that for electrons the probability saturates at high bias voltage but for holes the probability remains linear.

Table 2: Mean free path of different wavelength photons in Silicon[3]

\begin{tabular}{lc} 
Colour(wavelength) & Mean free path \\
\hline Blue(470nm) & $0.6 \mu \mathrm{m}$ \\
Green(525nm) & $1.2 \mu \mathrm{m}$ \\
Yellow(590nm) & $2.2 \mu \mathrm{m}$ \\
Red(625nm) & $2.9 \mu \mathrm{m}$
\end{tabular}

by electrons and holes, then it will be possible to probe this effect by comparing the PDE dependence on bias voltage using different wavelengths of light. The experimental method described in the following section was used to investigate this effect.

\section{Experimental Setup \& Analysis}

A schematic of the experimental setup is shown in Figure 4. Two MPPCs, a "data MPPC" and "reference MPPC", and an LED are placed inside a lightproof temperature-controlled chamber. The data MPPC is used for collecting PDE data, and is connected to a variable bias volt- 
age. The reference MPPC is used to normalise the light output of the LED, and is connected to a constant bias voltage. The LED and a diffuser are placed in front of the two MPPCs. The LED can be changed in order to measure the PDE for different light wavelengths. The four LEDs used are NICHIA NSPB500AS (Blue), NSPG510AS (Green), NSPY800AS (Yellow) and NSPR800AS (Red). The peak emission wavelengths of these LEDs are $470 \mathrm{~nm}, 525 \mathrm{~nm}$, $590 \mathrm{~nm}$ and $625 \mathrm{~nm}$ respectively. A $5 \mathrm{kHz}$ clock drives the LED and a gate generator for the ADC. The signals from the data and reference MPPCs undergo 100x amplification and are chargeintegrated over a 200ns gate by an ADC.

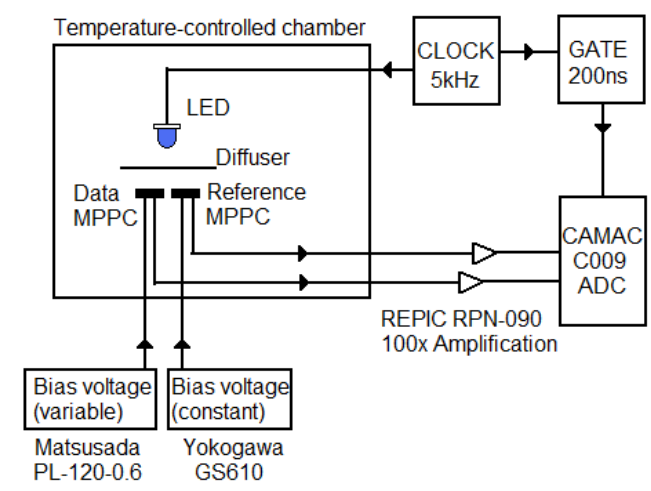

Figure 4: Schematic of experimental setup used to measure relative PDE.

During measurement, the temperature chamber is kept constant at $25^{\circ} \mathrm{C}$. The bias voltage of the data MPPC is scanned from 68.5 to $70.5 \mathrm{~V}$ in $0.05 \mathrm{~V}$ steps, corresponding to $\Delta V$ around $0.5 \mathrm{~V}$ to $2.5 \mathrm{~V}$, while the bias voltage of the reference MPPC is kept constant. At each voltage, $10^{5}$ events are taken, and this process is repeated with each of the four LED wavelengths. In addition, a measurement is taken at each voltage with the LED off, referred to as "noise data", in order to measure the dark noise rate. Measurements for several different MPPCs are made by changing the data MPPC. The reference MPPC, a T2K type, is kept the same in all measurements.

A typical ADC distribution is shown in Figure 5. The pedestal and 1 p.e. peaks are each fitted with Gaussian distribution. The gain is calculated using the separation of the two peaks. The ADC histogram is also integrated as shown in Figure 5, which gives the number of pedestal events $n_{p e d}$. This operation is performed for both the data and reference MPPCs with the LED on, and for the data MPPC with the LED off.

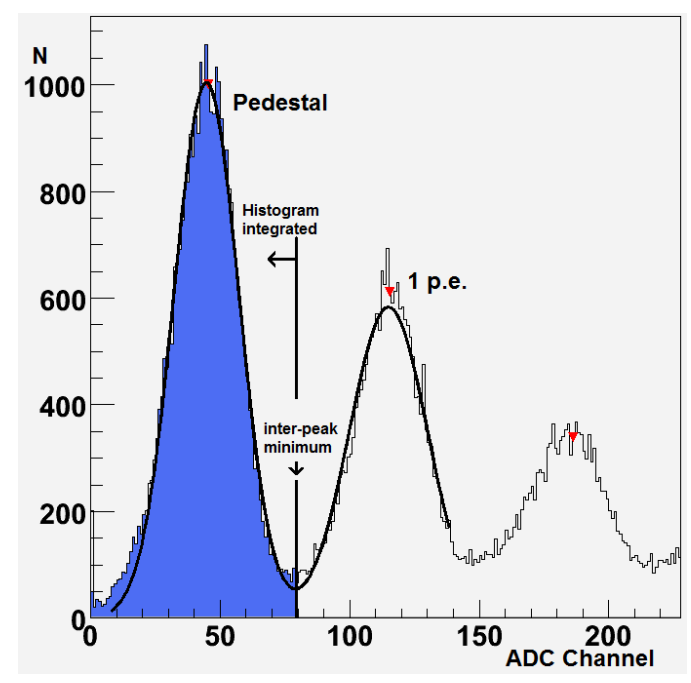

Figure 5: Typical ADC distribution from Data MPPC with LED on, showing pedestal and 1 p.e. peaks fitted with Gaussian distributions. The histogram is integrated from zero to the inter-peak minimum of the Gaussian fit between the pedestal and 1 p.e. peaks.

The gain is plotted against bias voltage and fit with a straight line. Extrapolating the line to the point where gain is zero gives the breakdown voltage $V_{b d}$ of the MPPC. This is used to determine the over-voltage $\Delta V$ of a particular measurement.

$$
\Delta V=V_{b i a s}-V_{b d}
$$

The PDE is calculated by treating the number of detected photoelectrons $n$ as a Poisson distribution with mean $\mu$. The probability of 
detecting $n$ photoelectrons is then

$$
P(n)=\frac{\mu^{n} \cdot e^{-\mu}}{n !} .
$$

The probability of detecting zero photons $P(0)$ can be expressed in terms of the number of pedestal events $n_{p e d}$ and total number of events $n_{\text {tot }}$ as

$$
P(0)=e^{-\mu}=\frac{n_{p e d}}{n_{\text {tot }}}
$$

and from this we can calculate the mean number of detected photoelectrons $\mu$

$$
\mu=-\ln \left(\frac{n_{p e d}}{n_{t o t}}\right)
$$

The presence of dark noise means that some events which should be in the pedestal are seen as 1 p.e. or higher events. This effect is corrected for as follows. The noise data taken from the data MPPC with the LED off is used. The number of noise events $n_{\text {noise }}$ at a given bias voltage is calculated as

$$
n_{\text {noise }}=n_{\text {tot }}^{\text {noisedata }}-n_{\text {ped }}^{\text {noisedata }}
$$

In order to correct a particular measurement for the effect of dark noise, the number of pedestal events which coincided with a noise event must be added to the original observed number of pedestal events. First $n_{\text {noise }}$ is scaled to the size of the pedestal to be corrected, using $n_{\text {ped }}$ and $n_{t o t}$ from the LED on data:

$$
n_{\text {noise }}^{\text {scaled }}=n_{\text {noise }} \times\left(\frac{n_{\text {ped }}}{n_{\text {tot }}-n_{\text {noise }}}\right)
$$

and then added to $n_{p e d}$ to give the corrected pedestal events $n_{\text {ped }}^{\text {corr }}$

$$
n_{\text {ped }}^{\text {corr }}=n_{\text {ped }}+n_{\text {noise }}^{\text {scaled }}
$$

The relative PDE is calculated using the fact that

$$
\mu=n_{\text {photons }} \times P D E
$$

and using $\mu$ of the reference MPPC measurement as a normalisation for the light output of the LED

$$
\text { relative } P D E=\frac{\mu(\text { data } M P P C)}{\mu(\text { ref } M P P C)}
$$

where

$$
\mu(\text { data } M P P C)=-\ln \left(\frac{n_{\text {ped }}^{\text {corr }}}{n_{\text {tot }}}\right)
$$

is the mean number of photoelectrons detected in the data MPPC and

$$
\mu(\text { ref } M P P C)=-\ln \left(\frac{n_{\text {ped }}}{n_{\text {tot }}}\right)
$$

is the mean number of photoelectrons detected in the reference MPPC and does not use the pedestal noise correction, since the reference MPPC is kept at constant bias voltage and thus the noise rate is a constant. Note that the calculation involves only the number of events in the pedestal peak, and therefore is not affected by crosstalk and afterpulsing in the MPPC. Finally, due to difference in the light output of different LEDs, the ratio of photons going to the data and reference MPPCs will change between measurements. To allow the different LED measurements to be compared, the relative PDE curves are normalised with each other such that the relative PDEs of all LED wavelengths are equal at some initial minimum value of $\Delta V$.

\section{Results}

The relative PDE calculated as in the previous section is plotted as a function of the overvoltage. The relative PDE against $\Delta V$ for two different T2K MPPCs and one Surface Mount MPPC are shown in Figure 6.The PDE curves have been normalised with each other such that the relative PDE is equal to unity at $\Delta V=0.9 \mathrm{~V}$. It can be seen that the PDE with blue light saturates at large $\Delta V$, and the PDE curves with green, yellow and red light saturate progressively less at large $\Delta V$. This effect is seen in all 3 measurements shown, and for both types of MPPC measured.

In an attempt to extract some quantitative information from these results, a fit was applied 

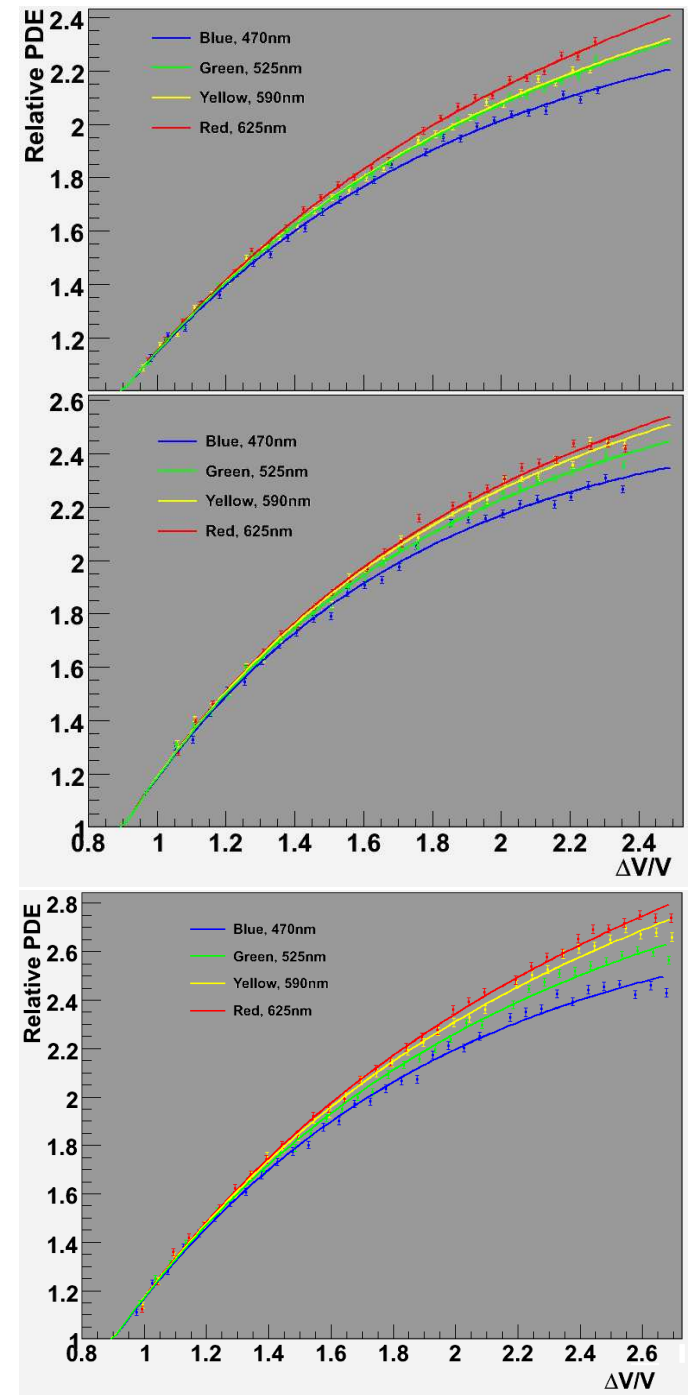

Figure 6: Relative PDE as a function of $\Delta V$. Top: T2K MPPC 1; Middle: T2K MPPC 2; Bottom: Surface Mount MPPC. At large $\Delta V$ there is a clear separation between the blue and red LED PDE curves. Note that the relative PDEs are all normalised such that the PDE at $\Delta V=0.9 \mathrm{~V}$ is unity.

according the the model outlined in section 2 . To model the electron Geiger probability an exponentially saturating function of the form

$$
\varepsilon_{\text {Geiger }}^{\text {electron }}=\varepsilon_{\max }\left(1-e^{-k\left(V-V_{0}\right)}\right)
$$

was used, and for the hole Geiger probability a linear function of the form

$$
\varepsilon_{\text {Geiger }}^{\text {hole }}=m\left(V-V_{0}\right)
$$

is used. By assuming a fraction $F$ of the light is absorbed in the p+ layer of the silicon, and that the remaining $(I-F)$ fraction of the light is absorbed in the $\mathrm{n}+$ layer, we obtain

$$
\varepsilon_{\text {Geiger }}^{\text {total }}=F \cdot \varepsilon_{\text {Geiger }}^{\text {electron }}+(1-F) \cdot \varepsilon_{\text {Geiger }}^{\text {hole }}
$$

In this way the varying bias voltage dependence of the PDE with light wavelength is modeled. In order to fit this function to the data, it is first assumed that for the blue LED measurement, $F=1$. This allows the parameters of $\varepsilon_{\text {Geiger }}^{\text {electron }}$ to be extracted by fitting to the blue LED data. Secondly, we assume that $m=2$ for $\varepsilon_{\text {Geiger }}^{\text {hole a }}$ value chosen by hand in order to match the result seen in Figure 3. The remaining green, yellow and red data are then fit using the parameters from the blue LED fit and only allowing $F$ to vary. This gives the fit values for $F$ seen in Table 3. In addition, the depth $d$ of the multiplication layer corresponding to a particular value of $F$ can be calculated using the attenuation length $\alpha$ for each wavelength of light.

$$
F=1-e^{-\alpha d}
$$

The resulting values of $d$ are also shown in Table 3 .

It can be seen that the value of $F$ decreases with increasing light wavelength, as predicted. However, while the estimated depth $d$ of the multiplication layer is of the right order, it gives inconsistent values for different measurements of the same MPPC, where $d$ should obviously not change with light wavelength. This suggests that the model is too simplistic at this stage to accurately describe the data.

\section{Conclusions \& Further Work}

The bias voltage dependence of the PDE of several MPPCs was measured with four different wavelengths of light. The results showed 
Table 3: Values of parameter $F$ and corresponding depth $d$ of multiplication layer extracted from fit of the relative PDE data. The value of $F$ decreases with increasing wavelength, as expected. For blue light, $F$ is manually fixed as 1 .

\begin{tabular}{llcc} 
& & $F$ & $d / \mu \mathrm{m}$ \\
\hline & Blue & 1 & - \\
T2K MPPC 1 & Green & 0.92 & 2.0 \\
& Yellow & 0.91 & 3.9 \\
& Red & 0.85 & 4.4 \\
\hline \hline \multirow{4}{*}{ T2K MPPC 2 } & Blue & 1 & - \\
& Green & 0.94 & 2.3 \\
& Yellow & 0.90 & 3.7 \\
& Red & 0.88 & 4.9 \\
\hline \hline \multirow{5}{*}{ Surf. Mount MPPC } & Blue & 1 & - \\
& Green & 0.93 & 2.1 \\
& Yellow & 0.87 & 3.3 \\
& Red & 0.84 & 4.2 \\
\hline
\end{tabular}

that for longer light wavelengths, the PDE saturated less at high over-voltage. This is in agreement with the model and simulation described in [2], in which the Geiger probability saturates at large bias voltage for electron triggered avalanches but remains linear at large $\Delta V$ for hole triggered avalanches. By fitting a simple model of this effect to the data, it was shown that the data is consistent with longer wavelengths of light penetrating more into the $n+$ silicon layer and therefore creating more hole triggered avalanches. There is therefore significant evidence that the Geiger probability of electron and hole triggered avalanches differs in their dependence on bias voltage. However, quantitative calculation of the depth of the multiplication layer based on the fit parameters gave inconsistent results. This leads to the conclusion that the model used to fit the data is not complete and needs improving.

Further work will involve improving the data set by making more measurements, and also by extending the wavelength range into infra-red and ultraviolet light. A quantitative analysis of the data will also be undertaken in greater detail. This is needed in order to fully understand and model the underlying effects which give rise to the observed change in bias voltage dependence.

\section{References}

[1] Hamamatsu Photonics K.K, MPPC Technical Information, http://www.hamamatsu.com

[2] H.Oide et. al., arXiv:0811.1402

[3] Virginia Semiconductor, Inc., Optical Properties of Silicon, http://www.virginiasemi.com/pdf/Optical Properties of Silicon71502.pdf 